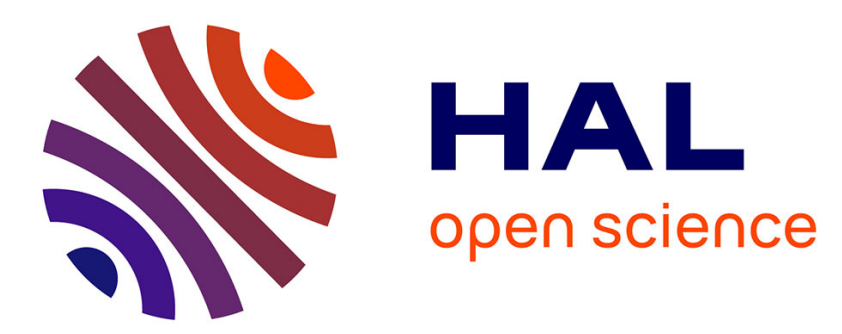

\title{
Sensitivity analysis for subsonic jet using adjoint of non local stability equations
}

Tobias Ansaldi, Christophe Airiau

\section{To cite this version:}

Tobias Ansaldi, Christophe Airiau. Sensitivity analysis for subsonic jet using adjoint of non local stability equations. AIAA 2015-2219, 21st AIAA/CEAS Aeroacoustics Conference, Jun 2015, Dallas, United States. pp.0, 10.2514/6.2015-2219 . hal-01661627

\section{HAL Id: hal-01661627 https://hal.science/hal-01661627}

Submitted on 12 Dec 2017

HAL is a multi-disciplinary open access archive for the deposit and dissemination of scientific research documents, whether they are published or not. The documents may come from teaching and research institutions in France or abroad, or from public or private research centers.
L'archive ouverte pluridisciplinaire HAL, est destinée au dépôt et à la diffusion de documents scientifiques de niveau recherche, publiés ou non, émanant des établissements d'enseignement et de recherche français ou étrangers, des laboratoires publics ou privés. 


\section{Open Archive TOULOUSE Archive Ouverte (OATAO)}

OATAO is an open access repository that collects the work of Toulouse researchers and makes it freely available over the web where possible.

This is an author-deposited version published in : http://oatao.univ-toulouse.fr/ Eprints ID : 18307

To link to this article : DOI:10.2514/6.2015-2219

URL : http://dx.doi.org/10.2514/6.2015-2219

To cite this version : Airiau, Christophe and Ansaldi, Tobias Sensitivity analysis for subsonic jet using adjoint of non local stability equations. (2015) In: AIAA 2015-2219, 21st AIAA/CEAS Aeroacoustics Conference, 22 June 2015 - 26 June 2015 (Dallas, United States).

Any correspondence concerning this service should be sent to the repository administrator: staff-oatao@,listes-diff.inp-toulouse.fr 


\title{
Sensitivity analysis for subsonic jet using adjoint of non local stability equations
}

\author{
Tobias Ansaldi* and Christophe Airiau ${ }^{\dagger}$ \\ Institut de Mécanique des Fluides de Toulouse, UMR 5502 CNRS/INPT-UPS, \\ Université de Toulouse Allée du Professeur Camille Soula, F31400 France
}

\begin{abstract}
Sensitivity analysis of some quadratic quantity related to acoustic waves with respect to any flow or wall disturbance is proposed in the configuration of subsonic jet flow. The generation of noise has been demonstrated to originate from convective instabilities that amplify in the jet stream. Several authors have investigated them through the Parabolized Stability Equations approach (PSE). The present work aims to develop the adjoint of the PSE to extract from a mathematically well posed problem the sensitivity coefficients which can be understood as gradient. The final objective is to propose some path of possible actuations in order to decrease noise emission in some jet flows. The shape and the location of the maximum of sensitivity are strongly related to the radial and streamwise variation of the base flow. In particular the maximum of sensitivity is located along the border of the potential cone and it seems to be well correlated with the location of the sound generation mechanism. In addition the sensitivity to axial momentum forcing is higher than to a radial momentum forcing. Finally the sensitivity increases when the perturbation is near to the exit of the nozzle.
\end{abstract}

\section{Introduction}

In the past many investigators ${ }^{1-6}$ have suggested based to theoretical and experimental results that flow instabilities is the dominant noise-generation mechanism for jet flows at high Mach number, at low Reynolds number and in perfectly expanded conditions. The problem is more complex in case of imperfectly expanded jet where shock-induced screech tone noise and shock-induced broad band noise ${ }^{8}$ have to be taken into account. More recent works ${ }^{9-11}$ have shown that instabilities can play an important role even for subsonic jet noise amplification. Furthermore, several experiments ${ }^{12-14}$ show that the dominant part of the turbulent mixing noise of high Reynolds number jets is generated by the large-scale structures of the turbulence as testament of the main role of instabilities. In fact the large-scale coherent structures are the instability waves of the jet, as many investigators ${ }^{6,15,16}$ have suggested.

In addition, a low computational cost model of the shear-layer instability modes in the co-axial jet based on the linear and non-linear Parabolized Stability Equation (PSE) has been developed and correlations between CFD results have been tested successfully $\left(\mathrm{see}^{2,4,5,11}\right)$. Thanks to the contribution of many investigators $^{11,17-19}$ it's now known that Parabolized Stability Equations are a powerful tool for the prediction of subsonic and supersonic jet noise .

The total flow field $\mathbf{Q}$, with components respectively velocity, density and pressure, is decomposed into a base flow $\mathbf{q}=\left(\underline{u}_{x}, \underline{u}_{r}, \underline{u}_{\theta}, \underline{\rho}, \underline{p}\right)^{t}$ and a small perturbation quantity $\mathbf{q}^{\prime}=\left(u_{x}^{\prime}, u_{r}^{\prime}, u_{\theta}^{\prime}, \rho^{\prime}, p^{\prime}\right)^{t}$. The superscript $t$ refers to the transpose of a matrix or a vector.

In the present works, a sensitivity analysis is performed for a subsonic low-Reynolds single stream jet. The goal is to identify the regions of the flow more sensitive to external perturbations in the momentum forcing and mass or heat injection.

By definition ${ }^{20}$ sensitivity is equivalent to a gradient of any functional or quadratic integral. This functional called $E$ could be a physical energy associated to the perturbed velocity, temperature and pressure.

*PhD student, Marie Curie Fellow, tobias.ansaldi@imft.fr

${ }^{\dagger}$ Professor, University of Toulouse, christophe.airiau@imft.fr 
Mathematically it can be written as a quadratic function of the full disturbance vector. In addition the energy can be defined globally, in the full computational domain or in a plane at a given $x$ streamwise location. So let define the local energy as :

$$
E_{x}(x)=\int_{0}^{\infty} \mathbf{q}^{\prime h} M \mathbf{q}^{\prime} m_{r} d r
$$

The superscript $h$ denotes transpose conjugate. $m_{r}$ is a metric and $M$ is a diagonal positive matrix which weights the component of the disturbance vector and it allows some various energy definitions. They are set respectively to the radius $r$ and to identity $I$ in the following.

The flow components are written in the cylindrical system of coordinates. We assume a axisymmetrical flow or a periodic perturbation in the azimuthal direction.

The global energy is defined from the local energy as:

$$
E=\frac{1}{2} \int_{x_{0}}^{x_{f}} E_{x}(x) d x
$$

where $x_{0}$ and $x_{f}$ are respectively the beginning and the end of the streamwise limit of the computational domain. Let keep the subscript 0 and $f$ to refer respectively to value at $x_{0}$ and $x_{f}$.

Sensitivity coefficients can be therefore explained as how the response of any variation in the output of a system expressed as a mathematical functional can be apportioned to different sources of variation in the input of the model. Such analysis is common in different fields of engineering and in the field of fluid dynamics since it is closely related to optimization problems and optimal control (Walther et al. ${ }^{21}$ and Airiau $^{22}$ ). In the last 45 years receptivity of boundary layers flows was investigated in different theoretical and computational manners. Recently, ${ }^{23}$ has demonstrated that receptivity coefficients and the approach based on adjoint equations ${ }^{22}$ can be associated to an optimization problem and therefore they were strongly closed to sensitivity coefficients. Later it was used by ${ }^{21,24}$ to perform optimal control in the laminar boundary layer flow. Sensitivity analysis based on the adjoint of compressible Navier-Stokes were also recently derived $^{20,25,26}$ and have led to some optimal control studies of the two dimensional shear layer in the aeroacoustic framework. Some other examples of sensitivity can be found in the mesh optimization and in the optimization of structures.

In the present work, the sensitivity of a quasi-3D jet flow is investigated on the base of adjoint of the Parabolized Stability Equations (APSE). In this paper results on an incompressible analytical unstable baseflow are presented and discussed. The same methodology will be coupled in the future to a LES solver where a mean flow will be determined first for single stream and later to dual stream flow.

The main objective is to investigate flow sensitivities to any disturbances and to define some new noise control strategies. The different steps of the methodology are briefly described in the Section I and II. Section III shows validation and results and a conclusion ended the paper.

\section{State equations : Parabolized Stability Equations}

The flow disturbances are considered as the state variables of the model and their evolutions are assumed to be well defined by solving the PSE equations.

The PSE were initially proposed by Herbert ${ }^{27}$ and some other authors during the same period ${ }^{28}$ to study the linear and non linear development of Tollmien-Schlichting waves in boundary layers. Later 2000, works from $^{8,29,30}$ have extended PSE for jet flow. The main advantages of the choice of PSE instead of Local Stability Theory (LST) are at least three:

- the small streamwise variations of the base flow and of the disturbances are directly taken into account in the formulation (contrarily to LST where local parallel flow is assumed).

- the eigenvalue problem no longer exists and the PSE is set of Partial Differential Equations (PDE) mostly parabolic in the streamwise direction

- since PSE are PDE, it is simpler to solve it by adding various boundary conditions and source terms. That means that they are used for receptivity and sensitivity analysis, in optimal flow control approaches and for weakly non-linear stability analysis 
In this paper, only linear PSE are considered, therefore the first step is the Linearized Euler equations for a compressible (axial-symmetric) flow in cylindrical coordinates $(x, r, \theta, t)$ written as:

$$
\left(B+A_{3} \frac{\partial}{\partial t}+A_{2} \frac{\partial}{\partial \theta}+A_{1} \frac{\partial}{\partial x}+A_{0} \frac{\partial}{\partial r}\right) \mathbf{q}^{\prime}=\mathbf{0} \Longrightarrow L_{L E E} \mathbf{q}^{\prime}=\mathbf{0}
$$

The viscosity is neglected here because its role is quite negligible in the aeroacoustic studies of jet flow and in the sound generation and propagation mechanisms. $A_{0}, A_{1}, A_{2}, A_{3}$ and $B$ are matrices function of the base flow quantities.

Each line of the matrix $L_{L E E}$ has been obtained from conservation equations:

$$
L_{L E E}=\left[\begin{array}{cc}
\text { continuity eq. } \\
\text { momentum eq. } & \text { for } r \\
\text { momentum eq. } & \text { for } \theta \\
\text { momentum eq. } & \text { for } x \\
\text { energy eq. } &
\end{array}\right]
$$

An important hypothesis is made at this step. It is assumed that the axial evolution of large scale structures is not subject to nonlinear interactions, but it is rather controlled by weakly non parallel mechanisms resulting from the divergence of the jet.

The usual PSE is a spatial stability approach. Assumption of small streamwise variations of the complex wave number is added, coming from the non local (also qualified as non-parallel) stability theory PSE developed by Herbert. ${ }^{31}$ Moreover, the streamwise slowly varying assumption implies that $\frac{\partial \mathbf{q}}{\partial x}$ and $\frac{d \alpha}{d x}$ are small and with viscosity that $\frac{\partial^{2} \mathbf{q}}{\partial^{2} x}$ and $\frac{d^{2} \alpha}{d^{2} x}$ are negligible.

Finally the disturbance quantities can be written in the quasi normal mode form as:

$$
\mathbf{q}^{\prime}(x, r, \theta, t)=\mathbf{q}(x, r) \chi(x) e^{i(m \theta-\omega t)}+c . c ., \quad \text { where } \quad \chi(x)=\exp \left[i \int_{x_{0}}^{x} \alpha(\xi) d \xi\right]
$$

In this equation $\mathbf{q}(x, r)$ is the shape function vector and it is assumed to be slowly varying in the $x$ direction, $\alpha(x)$ is the axial (streamwise) wavenumber which is a complex function of the only streamwise variable, $m$ is the fixed integer azimuthal wavenumber and the real number $\omega$ is the fixed angular frequency of the disturbance. $\hat{\mathbf{q}}(x, r)=\chi(x) \mathbf{q}(x, r)$ contains the full $x$-dependence of the perturbation.

Substituting Eq. 2 in Eq. 1 we obtain the main part of the parabolic equations called PSE for "Parabolized Stability Equations". The question of keeping the term $\frac{\partial p}{\partial x}$ in the equation which let some ellipticity ${ }^{31}$ is not discussed there. Finally a new set of PDE equations can be written:

$$
L_{P S E} \mathbf{q}=\mathbf{0}, \quad \text { with } \quad L_{P S E}=i \alpha A_{1}+i m A_{2}+i \omega A_{3}+B+A_{1} \frac{\partial}{\partial x}+A_{0} \frac{\partial}{\partial r}
$$

As usual in the stability problem the velocity disturbance is assumed to goes to zero when $r$ goes to infinity and following ${ }^{2,4}$ some specific boundary conditions are set on the axis.

By observing the decomposition of equation 2 it can be noticed that the streamwise change of the disturbance $\hat{\mathbf{q}}(x, r)$ can be described by the product of the shape function $\mathbf{q}(x, r)$ and of the exponential term $\chi(x)$. This ambiguity must be resolved by the introduction of an additional equation, called normalization or closure relation which imposes that the exponential growth of the disturbance is absorbed by the wave function part of the decomposition $\chi(x)$, making sure that the shape function $\mathbf{q}(x, r)$ stays slowly varying in $x$. The definition of the normalization is based on the definition of the complex wave number respectively in the local and non local approach and with any component of the perturbation $q_{k}^{\prime}$ :

$$
-i \frac{\partial \ln \left(q_{k}^{\prime}\right)}{\partial x}=\alpha_{\text {local }} \quad \text { and } \quad-i \frac{\partial \ln \left(q_{k}^{\prime}\right)}{\partial x}=\alpha_{P S E}-i \frac{1}{q_{k}} \frac{\partial q_{k}}{\partial x}
$$

Naturally in the local stability theory the wave number is independent of radial direction $r$ contrarily to the PSE theory case if the previous definition is kept. To remove this apparent dependency in $r$ we introduce a weighting in the definition of the complex wave number as following: 


$$
-i \int_{0}^{\infty}\left|q_{k}\right|^{2} \frac{\partial \ln \left(q_{k}^{\prime}\right)}{\partial x} m_{r} d r=\alpha(x)\left[\int_{0}^{\infty}\left|q_{k}\right|^{2} m_{r} d r\right]-i \int_{0}^{\infty} \bar{q}_{k} \frac{\partial q_{k}}{\partial x} m_{r} d r
$$

Where over-bar denotes complex conjugate and $m_{r}$ generally is a metric equal to 1 or $r$. Imposing the following condition is just a way to retrieve for $\alpha(x)$ the same definition as in the local stability theory:

$$
\int_{0}^{\infty} \bar{q}_{k} \frac{\partial q_{k}}{\partial x} m_{r} d r=0 \quad \text { and more generally } \quad \mathcal{N}(\mathbf{q})=\int_{0}^{\infty}(N \overline{\mathbf{q}})^{t} \frac{\partial N \mathbf{q}}{\partial x} m_{r} d r=0
$$

The matrix $N$ can let choose which components of the state vector are used in the closure relation. The choice of another specific normalization would not change the value of the physical disturbance, as soon as this normalization removes the waviness and growth of the disturbance from the shape function to include it in the exponential term as ${ }^{4,28,31}$ have shown.

The system with the unknown $(\mathbf{q}, \alpha)$ is only quasi-parabolic because a residual ellipticity due to the normalization condition and a streamwise pressure gradient term remains, see Airiau and Casalis, ${ }^{28}$ and Andersson et al. ${ }^{32}$ It is solved numerically using a streamwise first-order marching solution starting to the initial condition at $x=x_{0}$ which is the solution of the local linear stability theory (LST). The radial direction is discretized using a multidomain spectral collocation approach. Following the pass work of Piot et al., ${ }^{4}$ Hankel-based boundary conditions are imposed at the top of the computational domain, $r=8 d$, where $d$ is the diameter of the nozzle. The discretized problem is solved iteratively in the streamwise direction for the shape function $\mathbf{q}$ at each axial location. The wavenumber $\alpha$ is updated at each iteration using a Newton-Raphson method.

In the present work the PSE code called 'Pasteq'2 has been designed, written and validated by the ONERA stability team. A comparison with the pioneer work of ${ }^{5}$ is shown in Fig. 1, where the base flow is the same used for the validation of the sensitivity code, Section IV. The Fig. 1 shows the axial evolution of the real and imaginary part of the streamwise wavenumber $\alpha$. The full line displays the results from 'Pasteq' code and circles come from Yen and Messersmith. ${ }^{5}$ The discrepancy between the computations close to the exit of the nozzle is due to different initial conditions. Indeed, as said before, in this work the PSE are initialized using LST.
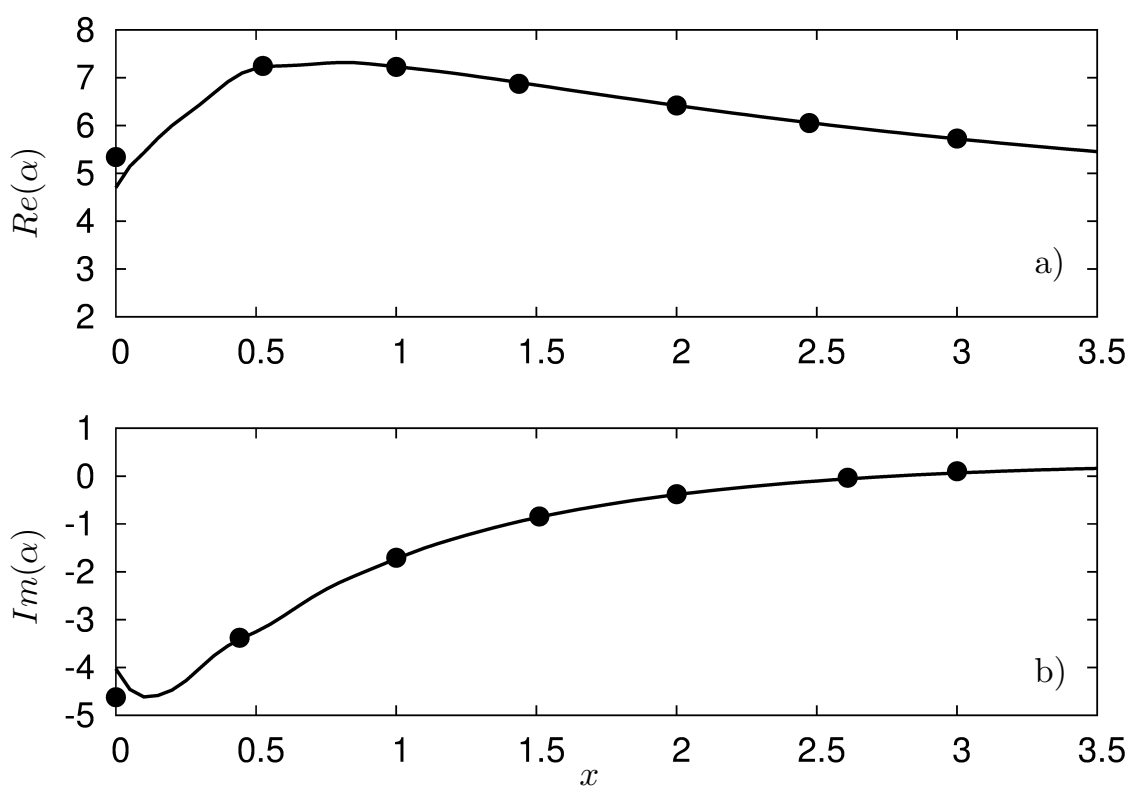

Figure 1. Comparison between present PSE and Yen et Messersmith PSE(•), 1998. a) Real part of $\alpha$, b) Imaginary part of $\alpha$ for a subsonic flow with $\omega=1.2 \pi$ and $m=0$. See also. ${ }^{5}$

\section{Sensitivity with Adjoint PSE}

In the following the sensitivity equations are derived in the cases where a small variation of the source forcing is applied as source term of the PSE as: 


$$
\chi(x) L_{P S E} \mathbf{q}(x, r)=\hat{\mathbf{f}}(x, r)=\chi(x) \mathbf{f}(x, r) \quad \text { and } \quad \int_{0}^{\infty} \mathbf{q}^{h} \frac{\partial \mathbf{q}}{\partial x} r d r=0
$$

As the initial state let us consider $\hat{\mathbf{f}}=0$ (no forcing).

The sensitivity $S_{\hat{f}_{k}}$ is therefore the gradient of $E$ with respect to any component of $\hat{\mathbf{f}}_{1}, \hat{f}_{k}$, translated mathematically as the relationship

$$
\delta E\left(\hat{f}_{k}\right)=\int_{\Omega} S_{\hat{f}_{k}} \delta \hat{f}_{k} d \Omega
$$

Where $\Omega$ is the computational domain.

Formally it can be written :

$$
S_{\hat{f}_{k}}=\nabla E_{\hat{f}_{k}}\left(\hat{f}_{k}=0\right)=\left(\frac{\partial E}{\partial \hat{f}_{k}}\right)_{\hat{f}_{k}=0}
$$

It is finally interpreted as how the variation in the output $E$ can be apportioned to variation in the input $\delta \hat{f}_{k}$ around the unforced condition. In case of flow control problem or optimization problem, the initial forcing $\hat{\mathbf{f}}$ is naturally non null.

To determine the sensitivity coefficient a Lagrangian functional is introduced:

$$
\mathcal{L}\left(\mathbf{q}, \hat{\mathbf{f}}, \alpha, \hat{\mathbf{q}}^{*}, n^{*}\right)=E-\left\langle\hat{\mathbf{q}}^{*}, \chi L_{P S E} \mathbf{q}-\hat{\mathbf{f}}\right\rangle_{\Omega}-\int_{0}^{\infty} \bar{n}^{*}\left\langle\mathbf{q}, \frac{\partial \mathbf{q}}{\partial x}\right\rangle_{r} d x+c . c .
$$

Where the brackets $\langle\mathbf{u}, \mathbf{v}\rangle_{\Omega}$ indicates an integral inner product in the complex plane defined by over the computational domain $\Omega$ :

$$
\langle\mathbf{u}, \mathbf{v}\rangle_{\Omega}=\int_{\Omega} \mathbf{u}^{h} \mathbf{v} d \Omega=\int_{x_{0}}^{x_{f}}\langle\mathbf{u}, \mathbf{v}\rangle_{r} d x, \quad \text { and } \quad\langle\mathbf{u}, \mathbf{v}\rangle_{r}=\int_{0}^{\infty} \mathbf{u}^{h} \mathbf{v} m_{r} d r
$$

In the previous equation we also consider the complex conjugate of the inner products to get real values.

The complex vectors $\hat{\mathbf{q}}^{*}(x, r)$ and $n^{*}(x)$ are some Lagrange multipliers (noted with a ${ }^{*}$ ) associated to the full PSE systems including the normalization condition. The complex vector $\hat{\mathbf{q}}^{*}(x, r)$ is more conveniently written in a manner similar to the direct variable $\mathbf{q}(x, r)$ by introduction of a wave-like part:

$$
\hat{\mathbf{q}}^{*}(x, r)=\mathbf{q}^{*}(x, r) \chi^{*}(x), \quad \chi^{*}(x)=\exp \left[i \int_{x_{f}}^{x} \bar{\alpha}(\xi) d \xi\right]
$$

It is easy to verify the following relationship with is often used later :

$$
\chi(x) \bar{\chi}^{*}(x)=\chi\left(x_{f}\right)=\chi_{f}=\text { constant }
$$

The normalization condition in the Lagrangian functional is an originality of this work and is necessary to get a mathematically well-posed problem.

Since the full PSE equations are equal to zero it is quite obvious that the variation of the Lagrangian functional is equal to the variation of the output quantity $E: \delta \mathcal{L}=\delta E$ and they have both the same gradient with respect to the state vector $\mathbf{q}$. Let us write formally the variation of the Lagrangian functional as :

$$
\delta \mathcal{L}=\frac{\partial \mathcal{L}}{\partial \mathbf{q}} \delta \mathbf{q}+\frac{\partial \mathcal{L}}{\partial \hat{\mathbf{f}}} \delta \hat{\mathbf{f}}+\frac{\partial \mathcal{L}}{\partial \mathbf{q}^{*}} \delta \mathbf{q}^{*}+\frac{\partial \mathcal{L}}{\partial \alpha} \delta \alpha+\frac{\partial \mathcal{L}}{\partial n^{*}} \delta n^{*}
$$

All the different directional derivatives given in Eq. 5 must vanish, except the term $\frac{\partial \mathcal{L}}{\partial \hat{\mathbf{f}}} \delta \hat{\mathbf{f}}$, indeed the sensitivity coefficient $S_{\hat{f}_{k}}$ is found as the factor term of $\delta \hat{f}_{k}$ :

$$
S_{\hat{f}_{k}}=\nabla \mathcal{L}_{\hat{f}_{k}}=\operatorname{Re}\left(\hat{q}_{k}^{*}\right)
$$

where $q_{k}^{*}$ is the $k$ component of the Lagrange multiplier vector $\mathbf{q}^{*}$. Imposing equal to zero the gradient with respect to the state vector lead to the so-called adjoint equations (APSE) where the adjoint state $\mathbf{q}^{*}$ is solution of : 


$$
\frac{\partial \mathcal{L}}{\partial \mathbf{q}} \delta \mathbf{q}=0
$$

Coupled with the new closing condition which could be referred as 'adjoint normalization':

$$
\frac{\partial \mathcal{L}}{\partial \alpha} \delta \alpha=0
$$

This adjoint closure relation can be reduced only to :

$$
\int_{0}^{\infty} \frac{\partial}{\partial x}\left(\mathbf{q}^{* h} A_{1} \mathbf{q}\right) m_{r} d r+\frac{\chi \bar{\chi}}{\chi_{f}} \int_{0}^{\infty} \mathbf{q}^{h} M \mathbf{q} m_{r} d r=0
$$

The other part leads after some calculations and integration by part to obtain the boundary conditions of the adjoint state $\mathbf{q}^{*}$ when $r \rightarrow \infty$ and to the so-called terminal condition of the adjoint problem. In fact, the adjoint equations are to be integrated upwind from $x_{f}$ to $x_{0}$. The initial condition of the adjoint problem is therefore a 'terminal' condition. Most detail are given in the appendix.

The calculation are quite close to those found $i^{21}$ where the adjoint equation where obtained for an optimal control of the boundary layer instabilities. Determining the terminal condition is quite complex, since all the equations have to be detailed. In this particular case where the output $E$ is defined in the whole computational domain, $\mathbf{q}^{*}\left(x_{f}, r\right)=0$ is the best solution.

Introducing a wall forcing instead of a source forcing will not change the methodology, and results can be found by adding some few developments in the previous equations as demonstrated in Airiau et al. ${ }^{24}$

\section{Validation and Results}

All the PSE and adjoint equations are solved with non-dimensional variable, as did in Piot et al. ${ }^{4}$ The subsonic base flow is determined from the analytical expression given firstly by Crow and Champagne ${ }^{33}$ and found as well in Piot et al. ${ }^{4}$ and Yen and Messersmith. ${ }^{5}$ The mean flow is given by:

$$
\begin{aligned}
\underline{u}_{x} & =\frac{1}{2}\left\{1+\tanh \left[\frac{1}{8 \Theta}\left(\frac{1}{2 r}-2 r\right)\right]\right\} \\
\Theta & =0.03 x+0.02
\end{aligned}
$$

The non-dimensional mean pressure and density are assumed uniform in the solution domain and respectively equal to $p=1 /\left(\gamma M^{2}\right)$ and $\rho=1$. The mean radial velocity $\underline{u}_{r}(x, r)$ is computed from the continuity equation. The computations were performed for Mach number $M=0.01$ with the axisymmetric instability mode, $m=0$ and a Strouhal number of $S t=0.6$.

The physical domain of interest and as well the computational domain starts at the nozzle exit, $x=0$.

A sixth order compact difference scheme ${ }^{34}$ is used in the radial direction. The streamwise derivative, $\left.\frac{\partial \mathbf{q}^{*}}{\partial x}\right|_{j}$, is approximated by the backward finite-difference form $\left(\mathbf{q}_{j+1}^{*}-\mathbf{q}_{j}^{*}\right) / \Delta x$ with integration from $x_{f}$ to $x_{0}$. The Eq. 6 and 7 are solved with a Newton-Raphson algorithm and convergence is fast, less than 10 iterations with $n^{*}$ for each streamwise location $x$. The iteration is repeated until a relative error smaller than $10^{-8}$. The state perturbation $\mathbf{q}(x)$ and the complex wave number $\alpha$ are required and have to be saved when running the PSE problem in the first step .

The APSE computations have been validated by following the steps outlined below:

- PSE code have been modified in order to solve:

$$
\chi L_{P S E} \mathbf{q}=\hat{\mathbf{f}}_{k}
$$

where $\hat{\mathbf{f}}_{k}$ is the vector with $f_{k}$ in the $k$-th position and zero otherwise.

- The variation of the quadratic function $\delta E$ is computed as a difference between Eq. 9 and Eq. 3, after two PSE runs:

$$
\delta E=E\left(\delta f_{k}\right)-E(0)
$$




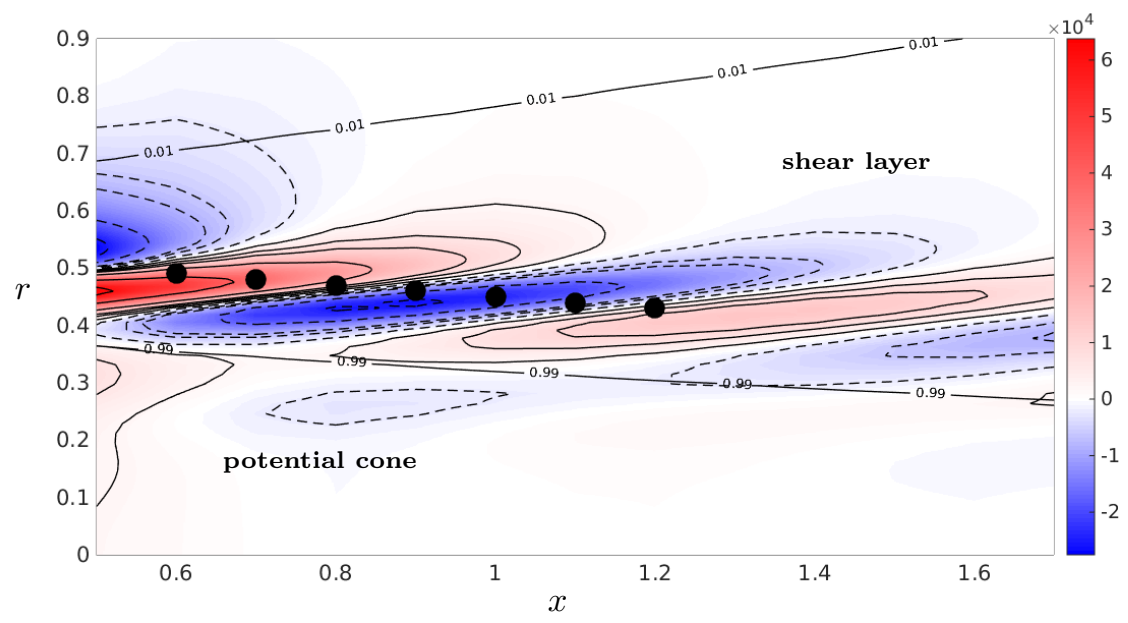

Figure 2. Spatial distribution of the gradient of $E$ with respect to a forcing acting in the x-momentum, $S_{f_{4}}$. Below the isoline $u_{x}=0.99 u_{x}(0, r)$ we can assume to be in the potential core otherwise the shear layer is delimited by the isoline $u_{x}=0.99 u_{x}(0, r)$ and the isoline $u_{x}=0.01 u_{x}(0, r)$. The isolines full line and dashed line indicates respectively positive and negative values of the sensitivity. With $\bullet$ are plotted the $(\tilde{x}, \tilde{r})$ used for validate de APSE.

- The variation of the quadratic function $\delta E$ is computed following APSE theory:

$$
\delta E=\int_{\Omega} \frac{\bar{\chi}\left(x_{f}\right)}{\bar{\chi}(x)} q_{k}^{*}(x, r) \delta f_{k}(x, r) d \Omega
$$

The small forcing $\delta f_{k}$ is chosen as:

$$
\delta f_{k}=\frac{\varepsilon}{k} F(x-\tilde{x}, r-\tilde{r}), \quad \text { with } \quad F(x-\tilde{x}, r-\tilde{r})=\exp \left(-\sigma_{x}(x-\tilde{x})^{2}-\sigma_{r}(r-\tilde{r})^{2}\right)
$$

Where $(\tilde{x}, \tilde{r})$ is the central location of the forcing and $k$ is a constant which normalizes $\delta f_{k}$ such that $\int_{\Omega} \delta f_{k} d \Omega=\varepsilon$. This Gaussian function, Eq. 12 is set in the $k$-th line of the Eq. 1 and it acts in a restricted region of the domain (see Fig. 2). Several tests have been done and the range of the different coefficients to define $f_{k}$ are given in Tab. 1.

\begin{tabular}{|c|c|c|c|c|}
\hline$\tilde{x}$ & $\tilde{r}$ & $\sigma_{x}$ & $\sigma_{r}$ & $\varepsilon$ \\
\hline \hline 0.6 & 0.49 & 50 & 50 & $10^{-2}$ \\
\hline 0.7 & 0.48 & 30 & 30 & $10^{-2}$ \\
\hline 0.8 & 0.47 & 30 & 30 & $10^{-2}$ \\
\hline 0.9 & 0.46 & 30 & 30 & $10^{-2}$ \\
\hline 1.0 & 0.45 & 30 & 30 & $10^{-2}$ \\
\hline 1.1 & 0.44 & 30 & 30 & $10^{-2}$ \\
\hline 1.2 & 0.43 & 30 & 30 & $10^{-2}$ \\
\hline
\end{tabular}

Table 1. Values of the coefficient used for the validation at different spatial position

Forcing smaller and more localized in the position nearest from the exit of the nozzle is required in order to avoid modifications of the initial condition $\mathbf{q}(0, r)$. The locations of the forcing have been chosen just out of the potential core where the sensitivity is high, see Fig. 2 and, because the arbitrary of the locations tested, they are simply placed along a straight line.

Results of Eq. 11 shown a very good agreement compared to the direct approach Eq. 10 for forcing acting in continuity, and axial-momentum equations, as displayed in Fig. 3. Similar results have been found for forcing acting in energy and r-momentum equations. 


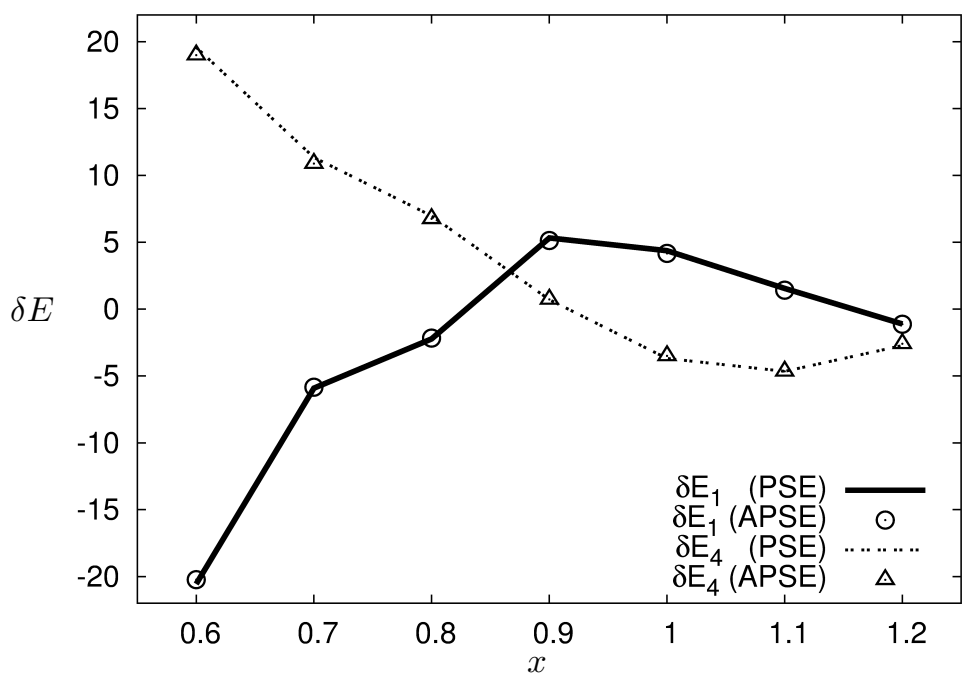

Figure 3. Comparison between results from Eq. 10 (lines) and Eq. 11 (symbols) is made.
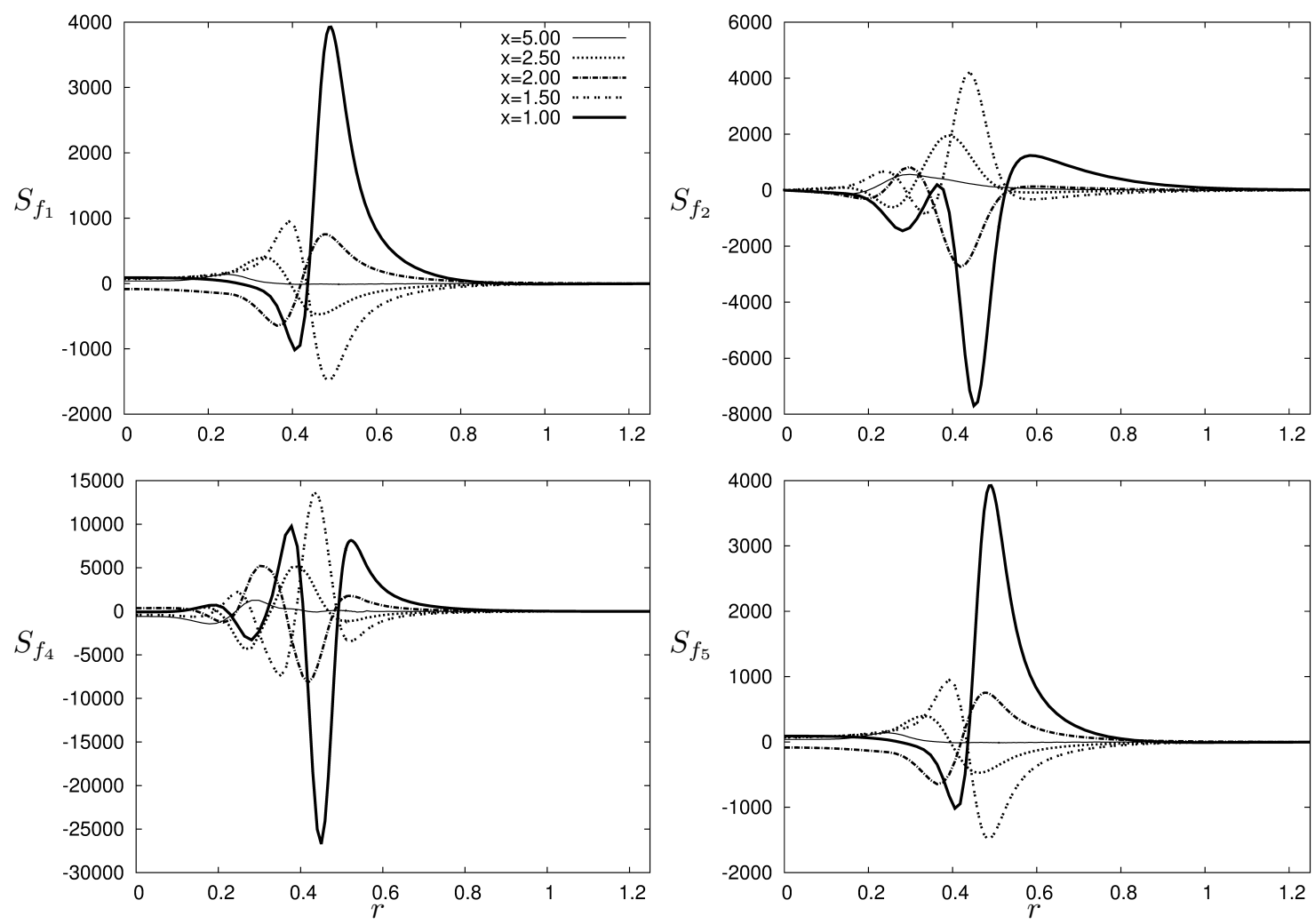

Figure 4. From top the bottom we have respectively, the gradient of $E$ with respect to the forcing acting in the continuity, $r$-momentum, $x$-momentum and energy equation at different fixed position in the stream-wise direction $(x=5.0,2.5,2.0,1.5,1.0)$ 
The variations of the total energy $E$ with respect to forcing acting in the continuity, momentum and energy equation are shown in Fig. 4.

It can be seen from Fig.4 that the shape of the sensitivity functions and the location of their maximum are strongly related to the radial and the streamwise variation of the base flow. In particular the maximum of sensitivity is located along the border of the potential cone and it is well correlate with the location of the sound generation mechanism. In addition the sensitivity increases when the streamwise coordinate decreases. That makes sense since it is natural to act as soon as possible on the noise generation mechanism if reduce noise emission is targeted. Another important point is that sensitivity to axial momentum forcing is much higher than to radial momentum forcing.

All these conclusions should be taken into account during the development of some noise reduction strategies. However it is also important to observe that the maximum of sensitivity, for each fixed position in $x$, is located near to a region where sensitivity is almost zero. Therefore the location of any control system must be very accurate to get some good efficiency.

\section{Conclusions}

As conclusion a first sensitivity model of adjoint PSE equations has been derived in the case of jet flow instability. The goal will be to investigate some new noise control strategy in a single and later in dual stream jet. First results concern a laminar flow but we currently couple the analysis stability and sensitivity with a base flow extracted from Large Eddy Simulations.

Another point will be in the near future to couple the stability and sensitivity solvers to a far-field sound propagation approach and to develop a sensitivity study for the full model (PSE + APSE + sound propagation).

As discussed in in $^{2,21,29}$ many questions remains relative to the location and the quality of the coupling between the PSE pressure disturbance and the far-field zone.

\section{Acknowledgments}

This work has been supported by the European Community as part of the FP7-PEOPLE-2012-ITN AeroTraNet 2. We would like to thanks J.P. Brazier and O. Léon from ONERA Toulouse for having provided the PSE code 'Pasteq'. This work was performed using HPC resources from CALMIP (grant 2014-24).

\section{Appendix}

Any field $\Phi(x, r, \theta, t)$ is decomposed into a base flow $\underline{\phi}(x, r)$ and a disturbance $\phi^{\prime}(x, r, \theta, t)$.

\section{A - PSE MATRICES}

All the values contained to the matrices are referred to the baseflow:

$$
A_{0}\left[\begin{array}{ccccc}
0 & \underline{\rho} & 0 & \underline{u}_{r} & 0 \\
0 & \underline{\rho}_{r} & 0 & 0 & 1 \\
0 & 0 & \underline{\rho}_{r} & 0 & 0 \\
\underline{\rho} \underline{u}_{r} & 0 & 0 & 0 & 0 \\
0 & 0 & 0 & -\underline{u}_{r} & \underline{\rho}_{r} \underline{u}_{r} M^{2}
\end{array}\right] \quad A_{1}=\left[\begin{array}{ccccc}
\underline{\rho} & 0 & 0 & \underline{u}_{x} & 0 \\
0 & \underline{\rho}_{x} & 0 & 0 & 0 \\
0 & 0 & \underline{\rho} \underline{u}_{x} & 0 & 0 \\
\underline{\rho}_{x} & 0 & 0 & 0 & 1 \\
0 & 0 & 0 & -\underline{u}_{x} & \underline{\rho}_{x} \underline{u}_{x}
\end{array}\right]
$$




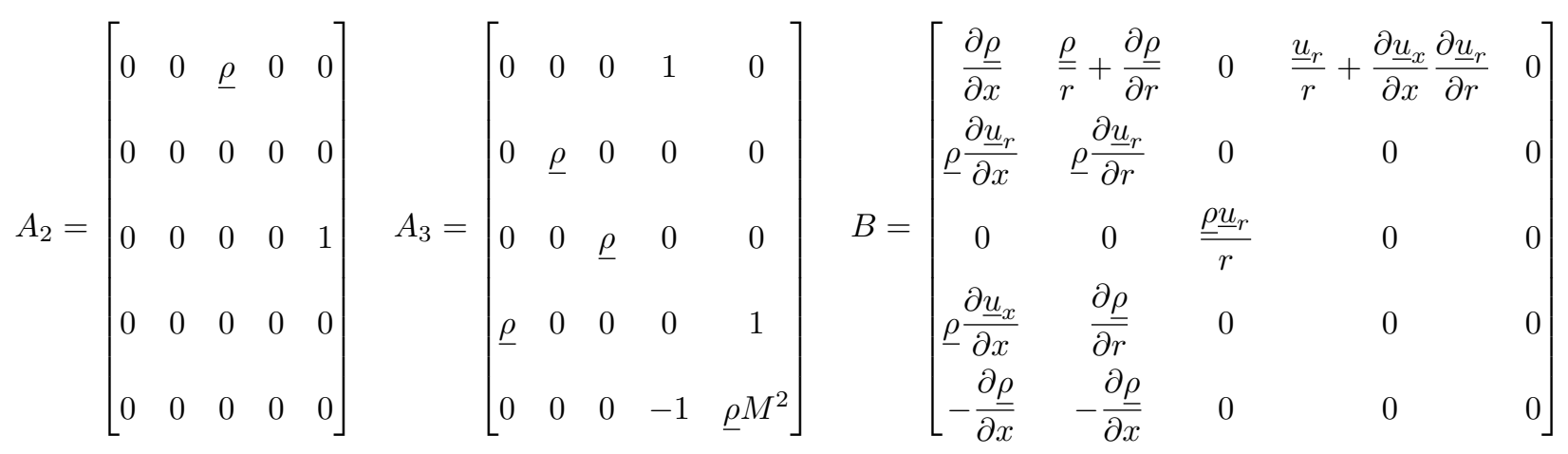

\section{B - APSE EQUATION}

\section{B-1 Procedure}

All the different directional derivatives vanish with exception of $\frac{\partial \mathcal{L}}{\partial \mathbf{f}} \delta \mathbf{f}$. It yields

$$
\begin{aligned}
\frac{\partial \mathcal{L}}{\partial \mathbf{q}} \delta \mathbf{q} & =\left\langle\chi_{f}\left(A^{t}+B^{t}-\frac{\partial A_{1}^{h}}{\partial x}-\frac{\partial A_{0}^{t}}{\partial r}-\frac{1}{r} A_{0}^{t}\right) \overline{\mathbf{q}}^{*}, \delta \mathbf{q}\right\rangle_{\Omega}-\left\langle\chi_{f} A_{1}^{t} \frac{\partial \overline{\mathbf{q}}^{*}}{\partial x}+\chi_{f} A_{0}^{t} \frac{\partial \overline{\mathbf{q}}^{*}}{\partial r}, \delta \mathbf{q}\right\rangle_{\Omega} \\
& +\left\langle n^{*} \frac{\partial \overline{\mathbf{q}}}{\partial x}-\frac{\partial\left(\bar{n}^{*} \overline{\mathbf{q}}\right)}{\partial x}, \delta \mathbf{q}\right\rangle_{\Omega}-2 \chi \bar{\chi}\langle\overline{\mathbf{q}}, \delta \mathbf{q}\rangle_{\Omega} \\
& +\left\langle\chi_{f} A_{1 f}^{T} \overline{\mathbf{q}}_{f}^{*}, \delta \mathbf{q}_{f}\right\rangle_{r}+\left\langle\bar{n}_{f}^{*} \overline{\mathbf{q}}_{f}, \delta \mathbf{q}_{f}\right\rangle_{r} \\
& +\left[\left\langle\chi_{f} A_{0}^{T} \overline{\mathbf{q}}^{*} r, \delta \mathbf{q}\right\rangle_{x}\right]_{r=0}+\left[\left\langle\chi_{f} A_{0}^{T} \overline{\mathbf{q}}^{*} r, \delta \mathbf{q}\right\rangle_{x}\right]_{r=\infty}+c . c .=0 \\
\frac{\partial \mathcal{L}}{\partial \alpha} \delta \alpha & =2 \int_{x_{0}}^{x_{f}}\left(E_{x} \int_{x_{0}}^{x} \delta \alpha d \xi\right) d x-\chi_{f} \int_{x_{0}}^{x_{f}}\left(\int_{0}^{\infty} \frac{\partial\left(\mathbf{q}^{* h} A_{1} \mathbf{q}\right)}{\partial x} r d r \int_{x_{0}}^{x} \delta \alpha d \xi\right) d x \\
& +\chi_{f}\left\langle\mathbf{q}_{f}^{*}, A_{1 f} \mathbf{q}_{f}\right\rangle_{r} \delta \alpha_{f}+\text { c.c. }=0
\end{aligned}
$$

with $A=i \alpha A_{1}+i m A_{2}-i \omega A_{3}$ and $\chi_{f}=\chi\left(x_{f}\right)=\bar{\chi}^{*}(x) \chi(x)$.

Imposing:

$$
\frac{\partial \mathcal{L}}{\partial \mathbf{q}^{*}} \delta \mathbf{q}^{*}=\mathbf{0} \quad \text { and } \quad \frac{\partial \mathcal{L}}{\partial n^{*}} \delta n^{*}=0
$$

we obtain respectively Eq. 3 and Eq. 4 . 


\section{B-2 Adjoint Parabolized Stability Equations}

Since all variations are arbitrary, except at boundaries where the conditions are fixed (such as, for example, at $\left.x=x_{0}\right)$.

- the different integrals vanish if the following Euler-Lagrange equations are satisfied:

$$
L_{P S E}^{*} \mathbf{q}^{*}=\mathbf{g}\left(\mathbf{q}, n^{*}\right)
$$

with

$$
L_{P S E}^{*}=-\frac{1}{r} A_{0}{ }^{h}+A^{h}+B^{h}-\frac{\partial A_{1}^{h}}{\partial x}-\frac{\partial A_{0}^{h}}{\partial r}-A_{1}^{h} \frac{\partial}{\partial x}-A_{0}^{h} \frac{\partial}{\partial r}
$$

and

$$
\mathbf{g}\left(\mathbf{q}, n^{*}\right)=\frac{1}{\bar{\chi}_{f}}\left[\left(n^{*}-\bar{n}^{*}\right) \frac{\partial \mathbf{q}}{\partial x}+\left(\frac{\partial n^{*}}{\partial x}+\chi \bar{\chi}\right) \mathbf{q}\right]
$$

- closing relation:

$$
i E_{x}+\int_{0}^{\infty}\left(\chi_{f} \frac{\partial\left(\overline{\mathbf{q}}^{* h} A_{1} \mathbf{q}\right)}{\partial x}\right) r d r+c . c .=0
$$

It is equation 7 .

- terminal conditions:

$$
\chi_{f} \int_{0}^{\infty} \overline{\mathbf{q}}_{f}^{* h} A_{0 f} \mathbf{q}_{f} r d r+c . c .=0 \quad \text { and } \quad \bar{\chi}_{f} A_{1 f}^{t} \mathbf{q}_{f}^{*}+n_{f}^{*} \mathbf{q}_{f}+\text { c.c. }=0
$$

- boundary condition:

$$
\left[\bar{\chi}_{f} r A_{0}^{t} \mathbf{q}^{*}\right]_{r=0}+\text { c.c. }=0 \quad \text { and } \quad\left[\bar{\chi}_{f} r A_{0}^{t} \mathbf{q}^{*}\right]_{r=\infty}+\text { c.c. }=0
$$

\section{References}

${ }^{1}$ Tam, C. K. W. \& Burton, D. E., "Sound generated by instability waves of supersonic flows. Part 1.. two-dimensional mixing layers," J. Fluid Mech., Vol. 138, 1984, pp. 249-271.

${ }^{2}$ Léon, O. \& Brazier, J.-P., "Application of Linear Parabolized Stability Equations to a Subsonic Coaxial Jet," AIAA/CEAS, 32nd Aeroacoustics Conference, 2011.

${ }^{3}$ Ray, P. K., Lawrence, C., Cheung, C., and Lele, S. K., "On the growth and propagation of linear instability waves in compressible turbulent jets," Phys. Fluids, Vol. 21, 2009.

${ }^{4}$ Piot, E., Casalis, G., Muller, F., and Bailly, C., "Investigation of the PSE approach for subsonic and supersonic hot jets. Detailed comparison with LES and Linearized Euler Equations results," aeroacoustic, Vol. 5.4 A VERIFIER, 2006 , pp. 361-393. 
${ }^{5}$ Yen, C. C. and Messersmith, N. L., "Application of Parabolized Stability Equations To the Prediction of jet Instabilities," AIAA/ASME, 36th Conference, 1998.

${ }^{6}$ Tam, C. K. W., "Directional acoustic radiation from a supersonic jet generated by shear layer instability," J. Fluid Mech., Vol. 46, No. 4, 1971, pp. 757-768.

${ }^{7}$ J. Fluid Mech., Vol. 565, 2006, pp. 197-226.

${ }^{8}$ Ray, P. K. and Lele, S. K., "Sound generated by instability wave/shock-cell interaction in supersonic jets," J. Fluid Mech., Vol. 587, 2007, pp. 173-215.

${ }^{9}$ Tam, C. K. W., Viswanathan, K., Ahuja, K. K., and Panda, J., "The sources of jet noise: experimental evidence," J. Fluid Mech., Vol. 615, 2008, pp. 253-292.

${ }^{10}$ Reba, R., Narayanan, S., and Colonius, T., "Wave-packet models for large-scale mixing noise," Int. J. Aeroacoustics, Vol. 9, No. 4, 2010, pp. 533-558.

${ }^{11}$ Ladeinde, F., Alabi, K., Colonius, T., Gudmundsson, K., Schlinker, R., and Reba, R., "An integrated RANS-PSE-wave packet tool for the prediction of subsonic and supersonic jet noise," No. AIAA 2010-4021, 2010.

${ }^{12}$ D. K. McLaughlin, G. L. M. and Toutt, T. R., "Reynolds Number Dependence in Supersonic Jet Noise," AIAA Journal, Vol. 15, 1977, pp. 526-532.

${ }^{13}$ D. K. McLaughlin, G. L. M. and Toutt, T. R., "Experiments on the Instability Waves in a Supersonic Jet and Their Acoustic radiation," J. Fluid Mech., Vol. 69, 1975, pp. 73-95.

${ }^{14}$ J. M. Seiner, T. R. S. B. and Ponton, M. K., "Reynolds Number Dependence in Supersonic Jet Noise," No. $15526-532$ in AIAA-paper, 1977.

${ }^{15}$ Morris, P. J. and Tam, C. K. W., "Near and far Field Noise for Large-Scale Instabilities of Axisymmetric Jets," No. 77-1351 in AIAA-paper, 1977.

${ }^{16}$ C. K. W. Tam, P. C. and Seiner, J. M., "Relationship Between Instability Waves and Noise of High-Speed Jets,", No. 91-0492 in AIAA-paper, 1991.

${ }^{17}$ Gudmundsson, K. and Colonius, T., "Parabolized Stability Equation Models for Turbulent Jets and Their Radiated Sound," No. AIAA 2009-3380, 2009.

${ }^{18}$ D. Rodríguez, A. Samanta, A. V. G. C. T. C. and Jordan, P., "Parabolized stability equation models for predicting large-scale mixing noise of turbulent round jets," No. AIAA 2011-2838, 2011.

${ }^{19}$ D. Rodríguez, A. Sinha, G. A. b. T. C., "Acoustic field associated with parabolized stability equation models in turbulent jets," No. AIAA 2013-2279, 2013.

${ }^{20}$ Pralits, J. O., Airiau, C., Hanifi, A., and Henningson, D. S., "Sensitivity Analysis Using Adjoint Parabolized Stability Equations for Compressible Flows," Flow Turbul. Combust., Vol. 65, No. 3/4, 2000, pp. 321-346.

${ }^{21}$ Walther, S., Airiau, C., and Bottaro, A., "Optimal control of Tollmien-Schlichting waves in a developing boundary layer," Phys. Fluids, Vol. 13, No. 7, 2001, pp. 2087-2096.

${ }^{22}$ Airiau, C., "Non-parallel acoustic receptivity of a Blasius boundary layer using an adjoint approach," Flow Turbul. Combust., Vol. 65, No. 3/4, 2000, pp. 347-367.

${ }^{23}$ Airiau, C., Walther, S., and Bottaro, A., "Boundary layer sensitivity and receptivity," C.R. Mecanique, Vol. 330, 2002, pp. 259-265.

${ }^{24}$ Airiau, C., Bottaro, A., Walther, S., and Legendre, D., "A Methodology for Optimal Laminar Flow Control : Application to the Damping of Tollmien-Schlichting Waves in a Boundary Layer," Phys. Fluids, Vol. 15, No. 5, 2003, pp. 1131-1145.

${ }^{25}$ Spagnoli, B. and Airiau, C., "Adjoint analysis for noise control in a two-dimensional compressible mixing layer," Computers and Fluids, Vol. 37, 2008, pp. 475-486.

${ }^{26}$ Wei, M. and Freund, J. B., "A noise-controlled free shear flow," J. Fluid Mech., Vol. 546, 2006, pp. $123-152$.

${ }^{27}$ Herbert, T., "Parabolized stability equations," AGARD REPORT, 1993.

${ }^{28}$ Airiau, C. and Casalis, G., "Boundary layer linear stability using a system of parabolic equations," Rech. Aérosp., Vol. 5, 1993, pp. 57-68.

${ }^{29}$ Cheung, L. C. and Lele, S. K., "Acoustic radiation from subsonic and supersonic mixing layers with nonlinear PSE," No. AIAA 2004-0363, 2004.

${ }^{30}$ Cheung, L. C., Bodony, D. J., and Lele, S. K., "Noise radiation predictions from jet instability waves using a hybrid nonlinear PSE-acoustic analogy approach," No. AIAA 2007-3638, 2007.

${ }^{31}$ Herbert, T., "Parabolized stability equations," Annu. Rev. Fluid Mech., Vol. 29, 1997, pp. 245-283.

${ }^{32}$ P. Andersson, D. H. and Hanifi, A., "On a stabilization procedure for the parabolic stability equations," Journal of Engineering Mathematics, Vol. 33, pp. 311.

${ }^{33}$ Crow, S. C. and Chamapagne, F. H., "Orderly structure in jet turbulence," J. Fluid Mech., Vol. 48, 1971, pp. 547-591.

${ }^{34}$ Lele, S. K., "Compact finite difference schemes with spectral-like resolution," J. Comput. Phys., Vol. 103, 1992, pp. 16-42. 